\title{
The Effects of Preliminary Training Conditions upon DRL Performance in the Hippocampectomized Rat'
}

\author{
LEONARD W. SCHMALTZ AND ROBERT L. ISAACSON \\ Department of Psychology, University of Michigan, Ann Arbor, Michigan 48104
}

(Received 8 October 1965)

\begin{abstract}
SCHMALTZ, L. W. AND R. L. IsAACSON. The effects of preliminary training conditions upon DRL perforance in them hippocampectomized rat. Physiol. Behav. 1 (2) 175-182, 1966.-Rats suffering radical bilateral hippocampal destruction, destruction of the posterolateral neocortex, and normal animals were trained on a DRL 20 reinforcement schedule in an operant conditioning situation. Half of the animals received prior training on a continuous reinforcement schedule while the other half did not. Hippocampectomized rats placed on the DRL 20 schedule without prior experience on CRF were found to bar press significantly more than control animals, but received as many reinforcements and achieved as high a percentage of reinforced responses as did these animals. Hippocampectomized rats given long experience on CRF before the DRL 20 schedule bar pressed significantly more, received fewer reinforcements, and achieved a lower percentage of reinforced responses than control animals. Control animals with partial neodecortication did not differ in performance from unoperated rats in either situation. Data are reported concerning the relationship of the brain lesions to acquisition of the bar press response.
\end{abstract}

Hippocampus DRL schedule of reinforcement Operant conditioning

CLARK and Isaacson [1] found that rats with bilateral hippocampal damage were impaired in their ability to acquire a DRL 20 (differential reinforcement of low rates of responding) schedule of reinforcement. Ellen, Wilson, and Powell [5], however, reported findings suggesting that bilateral hippocampal lesions do not produce impairment in the rat's ability to acquire the same schedule of reinforcement.

The present study was undertaken to replicate the Clark and Isaacson findings and to answer a basic question which their work left unanswered. The deficits in the performance of their hippocampectomized animals could have been due to their inability to inhibit the bar press response for the required $20 \mathrm{sec}$ or to defective temporal discriminatory ability. There are, of course, other possible explanations; but these two views seem to best account for the behavior of hippocampectomized rats on a DRL schedule.

If hippocampal rats are significantly impaired in their temporal discriminatory ability, they should not be able to learn the DRL 20 schedule under any acquisition conditions. The present work explored the possibility that animals with bilateral hippocampal damage can master the DRL 20 schedule if the acquisition conditions are such that long experience on CRF (continuous reinforcement) does not precede the shift to the DRL schedule. A preliminary study indicated that such was the case.

Clark and Isaacson [1] also state it was their impression that hippocampal rats were easier to shape initially to the bar press response. This finding, though subjective, is in accordance with observations that hippocampal rats appear to acquire certain simple behavior more rapidly than normal rats or rats with neocortical damage $[6,7,17]$. An objective measure of shaping was developed to allow evaluation of the impression that hippocampal rats were easier to shape than normal animals.

\section{METHOD}

Subjects were forty male Long-Evans hooded rats approximately 120 days old at the start of the experiment. Fourteen of the animals received bilateral hippocampal destruction, 14 received lesions of the cortex overlying the hippocampus, and 12 served as unoperated controls.

Clean surgical technique was used. Subjects were anesthetized with sodium pentobarbital; they were not held in a stereotaxic instrument during the operation. The general operative procedure has been described before [6]. In the experimental group, the hippocampus was first exposed and then removed by aspiration. In making neocortical lesions, the hippocampus was similarly exposed but left intact. After bleeding had ceased, Gelfoam was inserted in the wound, the temporal muscles replaced and the scalp closed. The animals received intramuscular injections of penicillin (Pronapen) Immediately before the operation and an antibiotic (Tetracyn) was added to their drinking water for three days postoperatively.

${ }^{1}$ This research was supported by Contract No. DA-MD-49-193-63-G120 from the Office of the Surgeon General, U.S. Army. 
A $23 \mathrm{hr}$ deprivation schedule was initiated for all animals two weeks before the shaping procedure began. The rats were allowed to eat powdered mash ad libitum for $1 \mathrm{hr}$ each day. At the end of the two week period, all animals' weights had stabilized at approximately 90 per cent of their preoperative or ad libitum weights.

All of the shaping was done by the same experimenter. One $45 \mathrm{mg}$ pellet was discharged into the food cup before the rat was put into the operant chamber (Grason-Stadler Model E3125A-300). Using the successive approximations technique, the rats were first magazine trained and then shaped to the bar press response. After each reinforcement. at least a $20 \mathrm{sec}$ interval had to elapse before another reinforcement was given. This was controlled automatically by the operant' apparatus. Even a bar press by the animal did not elicit a pellet if it came within the $20 \mathrm{sec}$ interval. The animal reached the shaping criterion when it had pushed the bar twice in $1 \mathrm{~min}$, and after each bar press had gone directly to the food cup for reinforcement. The two responses did not have to be reinforced ones.

If the animal had not been shaped after an initial $45 \mathrm{~min}$, it was removed from the operant chamber. One hour later, the rat was given ad libitum food for $1 \mathrm{hr}$. An animal was given daily $45 \mathrm{~min}$ shaping sessions until it met the behavioral criterion. During the shaping sessions, all animals were $21.5 \mathrm{hr}$ food deprived.

The number of minutes in the operant chamber and the number of reinforcements needed to reach the shaping criterion were recorded for each animal.

After reaching the shaping criterion, an animal was given an additional $30 \mathrm{~min}$ in the operant chamber regardless of the point in the 45 min session in which shaping took place. This $30 \mathrm{~min}$ session was recorded as the first experimental session.

Experimental sessions were of two types. Animals in Condition I were placed on continuous reinforcement. Those in Condition II were placed on the DRL 20 schedule of reinforcement. There were 6 unoperated rats, 7 partially neodecorticate animals, and 7 with bilateral hippocampal damage in each Condition. One hour after each $30 \mathrm{~min}$ experimental session, the animals were given ad libitum food for $1 \mathrm{hr}$. They were then deprived for $21.5 \mathrm{hr}$ until the next experimental session.

The animals in Condition I were run for 20 consecutive continuous reinforcement sessions. During this time, the number of bar presses per session was recorded for each rat. On the twenty-first day, Condition I animals were switched to a DRL 20 schedule. They were given a total of $16 \mathrm{con}$ secutive DRL 20 sessions. For each session, the total number of bar presses and the number of reinforced responses was recorded for each animal.

Condition II anintals received 36 consecutive DRL 20 sessions. As in Condition I, the total number of bar presses and the number of reinforced responses was recorded for each animal.

\section{HISTOLOGICAL EVALUATION}

Following the experiment, all lesioned animals were sacrificed by an overdose of sodium pentobarbital, intracardially perfused with $10 \%$ formalin, and the brains removed. Following infiltration and embedding with paraffin, serial sections between 15-20 $\mu$ thickness were made throughout the lesioned areas. As a general procedure, every tenth section was retained. stained with thionin, and mounted.
The lesion produced in each brain was reconstructed on representative diagrams of the rat brain adapted from the atlas by DeGroot [2] at regularly spaced levels throughout the lesioned area. Superimposed on these representative diagrams were $0.5 \mathrm{~cm}$ squares. By counting the number of squares covered by each lesion, it was possible to compute a numerical index of the amount of total brain damage sustained by the animal and the extent of damage to specific structures within the brain.

In addition, drawings of the maxmal and minimal extent of the lesions were prepared for the hippocampal and neodecorticate animals from the individual reconstructions, In so doing the largest lesion on each side of the brain at each of the seven representative sections and the smallest lesion on each side of the brain at the same levels were combined onto the same drawings. These are presented in Figs. 1 and 2 .

In general, brain damage in the lesioned aninals was limited to four structures-the posterior neocortex. the corpus callosum, the fimbria, and the hippocampus.

The 14 animals in the neodecorticate group were found to have sustained a mean of 861.8 units $(0.5 \mathrm{~cm}$ squares $)$ of neocortical damage. The mean damage to the corpus callosum was 176.6 units. Only 4 of the 14 animals in this group sustained any damage to the fimbria. In all cases the damage was slight, the mean danuge being only 8.0 units of a possible 360 units. The total mean brain damage done to these animals was 1046.4 units.

Similar scores were computed for the animals receiving bilateral hippocampal lesions. It must. however, be mentioned at this time that two of the animals in this group suffered extensive damage to several thalamic nuclei, and, in one case, to the lateral septal area. These two animals, which were in Condition II, differed significantly in performance from the other hippocampal animals in this condition and they were excluded from the study. Their performances and a description of the damage sustained will be included in the discussion section, below.

The remaining twelve animals in the hippocampal group sustained the following mean amounts of damage: to the neocortex-801.8 units, to the corpus callosum- -273.4 units, to the fimbria-273.4 units, to the hippocampus-813.6 units; the mean total damage was $2,162.2$ units.

It was possible using the unit technique to compute the percentage of hippocampus destroyed for each animal. To obtain the percentage, the number of units destroyed was divided by the total number of squares (units) which the hippocampus encompassed on the seven representative diagrams. Using this technique, the percentage of hippocampus destroyed in the 12 animals varied from 54 per cent to 84 per cent. The mean percentage of hippocampal destruction was 59 per cent.

It was stated earlier that the brain damaged animals were divided into Conditions I and II. In the case of both the hippocampal and neodecorticate animals, there were no great differences in the amount of brain damage sustained by animals in the two Conditions.

Gliosis was observed in the thalamus in a number of animals in both the hippocampally ablated and the neodecorticate groups. This involved the lateral nucleus primarily. In no case was it extensive and was not, as far as could be determined, caused by direct damage to the thalamus. Most likely it resulted from surgically induced infarction of blood vessels in the region. A comparison of the behavior of the animals with and without thalamic gliosis did not suggest any effect 

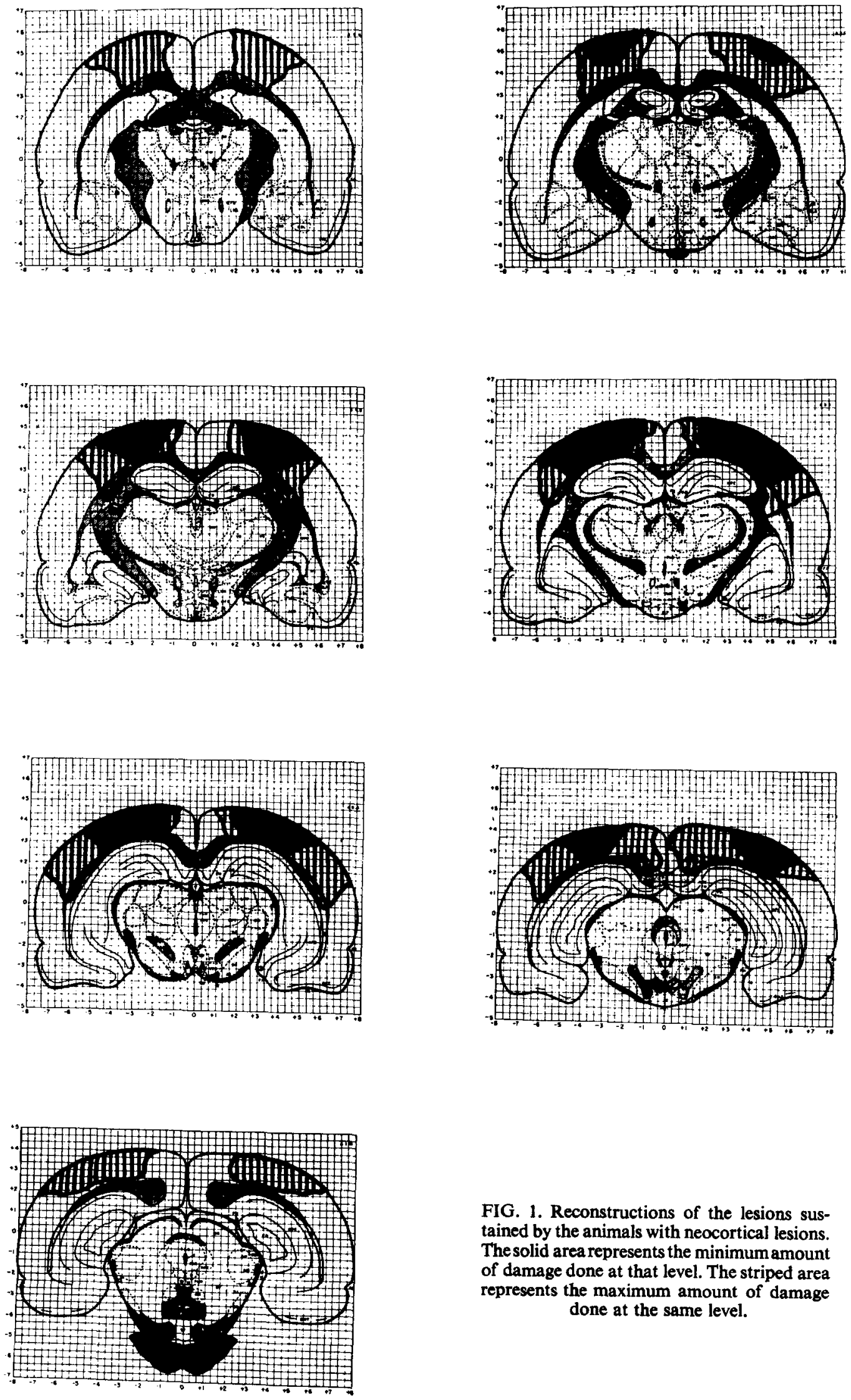

FIG. 1. Reconstructions of the lesions sustained by the animals with neocortical lesions. The solid area represents the minimum amount of damage done at that level. The striped area represents the maximum amount of damage done at the same level. 

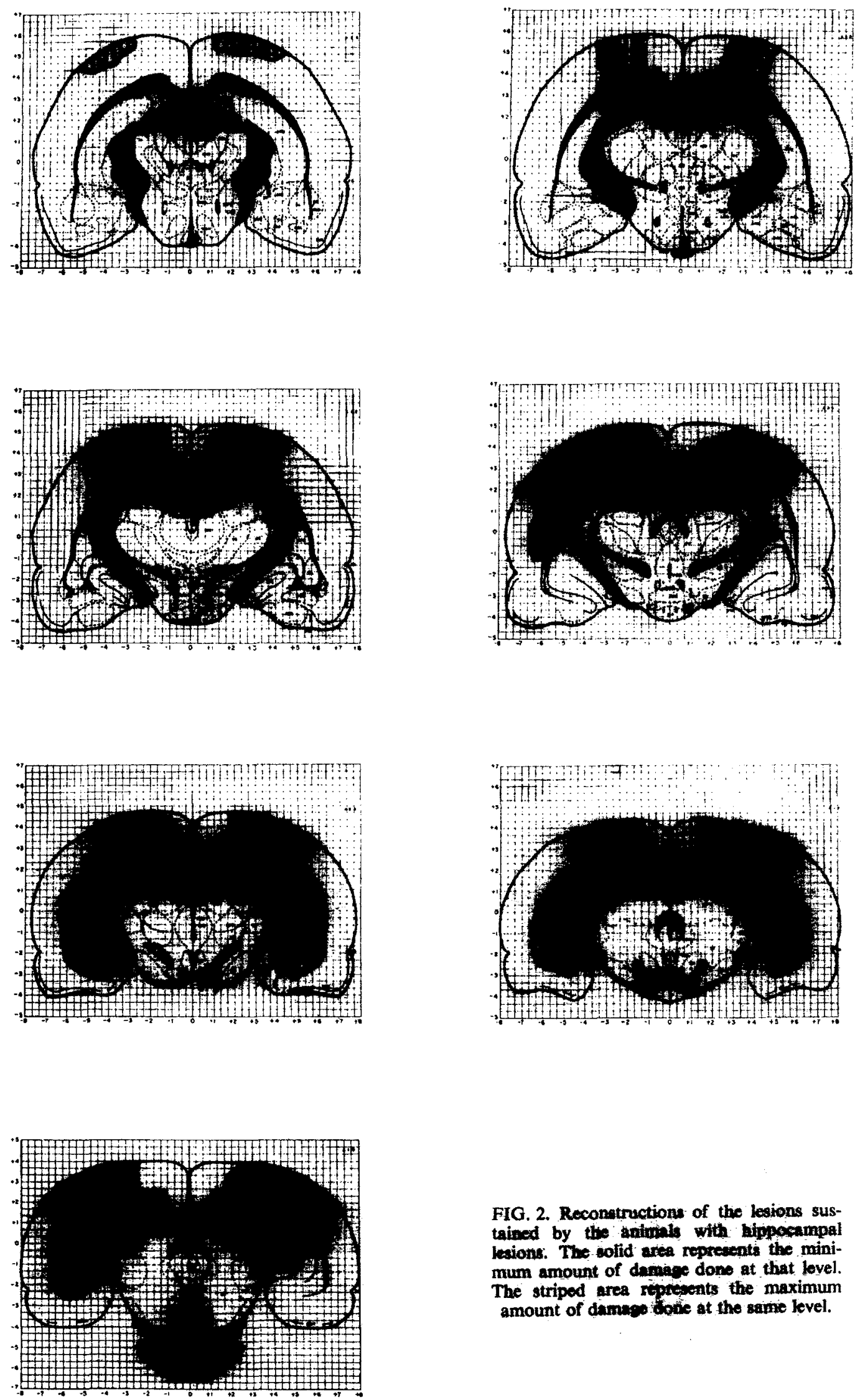

FIG. 2 Recontruction of the lesions sustained by the enituils with bippoemmal lesions. The solid area represents the minimum amount of damage done at that lovel. The striped area rioptesents the maximum amount of damios tote at the same kvel. 
of the gliosis. Clark and Isaacson [1] reported similar thalamic gliosis in several of their hippocampal animals and also did not find any effect on DRL performance.

\section{RESULTS}

Shaping. Graphs showing the shaping results for all three groups of animals are given in Fig. 3.

The results are in the expected direction in that the hippocampal animals spent less time in the operant chamber (median test: $\chi^{2}=4.16 ; p<0.05$ ) and required fewer reinforcements (median test: $\chi^{2}=4.16 ; p<0.05$ ) than did the unoperated normal animals to reach the shaping criterion.

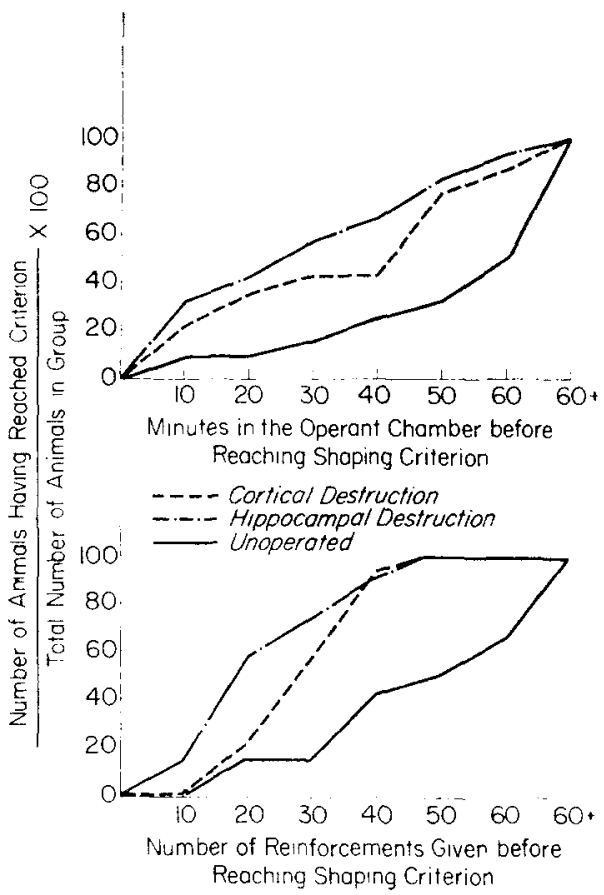

FIG. 3. Cumulative percentage curves for the two measures of speed of shaping.

Using the same median test, comparisons between the normal animals and the neodecorticate animals in both time spent in the operant chamber and number of reinforcements given before reaching the shaping criterion did not produce statistical significance. The same was true of similar comparisons between the neodecorticate rats and the hippocampal rats.

Pearson product-moment correlation coefficients were computed between total units of brain damage and speed of shaping within both the neodecorticate and hippocampal groups. There was no significant relation, as reflected by the correlation coefficients, between total units of brain damage, unit damage to neocortex, corpus callosum, fimbria, or hippocampus and number of reinforcements necessary or time spent in the operant chamber before reaching the shaping criterion within either group.

Condition I: CRF performance. The animals in this condition were given 20 daily CRF sessions of 30 min duration before the shift to the DRL 20 schedule took place. During this time, there was no statistically significant difference in the number of bar press responses given by the three groups of animals. A two-way analysis of variance showed no effect among groups of animals or over CRF sessions. There was also no significant interaction effect.

Condition I: DRL 20 performance. Once the three groups of animals had been placed on the DRL 20 schedule, differences among them were found. These can be seen in Fig. 4.
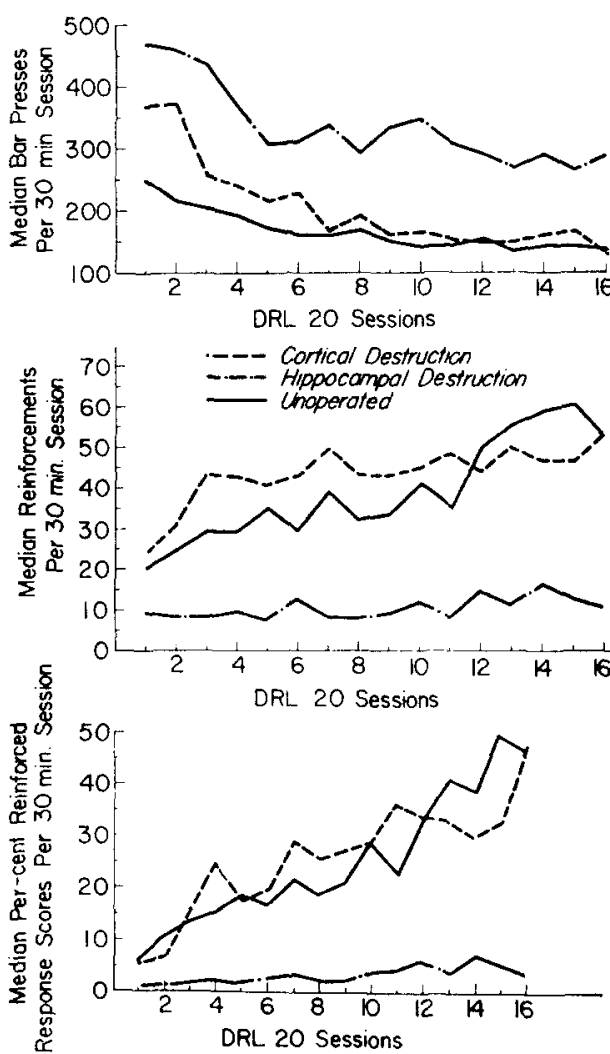

FIG. 4. Performance measures for the animals in Condition I on DRL 20. (The animals in this Condition had previous experience on CRF.)

A two-way analysis of variance showed a statistically significant effect among the three groups of animals in number of bar presses on the DRL schedule $(F=12.10 ; p<0.01)$. There was also a significant effect over DRL sessions $(F=$ $19.25 ; p<0.01)$ and a significant interaction effect $(F=$ $2.19 ; p<0.01$ ). Individual comparisons of the three groups of animals showed that the hippocampectomized rats bar pressed significantly more $(\mathrm{F}=14.19 ; p<0.01)$ than did the cortical control animals. There was no statistically significant difference between the cortical control animals and the unoperated rats.

A two-way analysis of variance was also computed for the number of reinforcements received by the three groups of animals while on the DRL 20 schedule. The analysis revealed significant effects across the groups of animals $(F=20.38$; $p<0.01)$ and over DRL sessions $(F=10.76 ; p<0.01)$. There was also a highly significant interaction effect $(\mathrm{F}=$ 409.54; $p<0.01$ ).

Individual comparisons showed that the animals with hippocampal damage received significantly fewer reinforce- 
ments $(F=36.19 ; p<0.01)$ than did the cortical control animals while on the DRL 20 schedule. There was no difference between the cortical control animals and the normal animals.

For each animal in Condition 1 , a per cent reinforced response score was computed for each session. This percentage was computed by dividing the number of reinforced responses during a session by the total number of responses made during the same session. Figure 4 presents graphically the median per cent reinforced response scores for each group of animals in Condition I for the 16 DRL 20 sessions.

For each of the lesioned animals in Condition I, three "performance scores" were computed. These were the means of the per cent reinforced response scores, the number of bar presses, and the number of reinforcements received averaged over the last $5 \mathrm{DRL}$ sessions. There was no significant correlation (Pearson product-moment) between any of these performance scores and total unit brain damage, unit damage to the neocortex, or unit damage to the corpus callosum within the neodecorticate group in Condition I. Within the hippocampectomized group in Condition I, there was no significant correlation (Pearson product-moment) between any of the three performance scores and total unit brain damage, unit damage to the hippocampus, unit damage to the neocortex, unit damage to the corpus callosum, or unit damage to the fimbria.
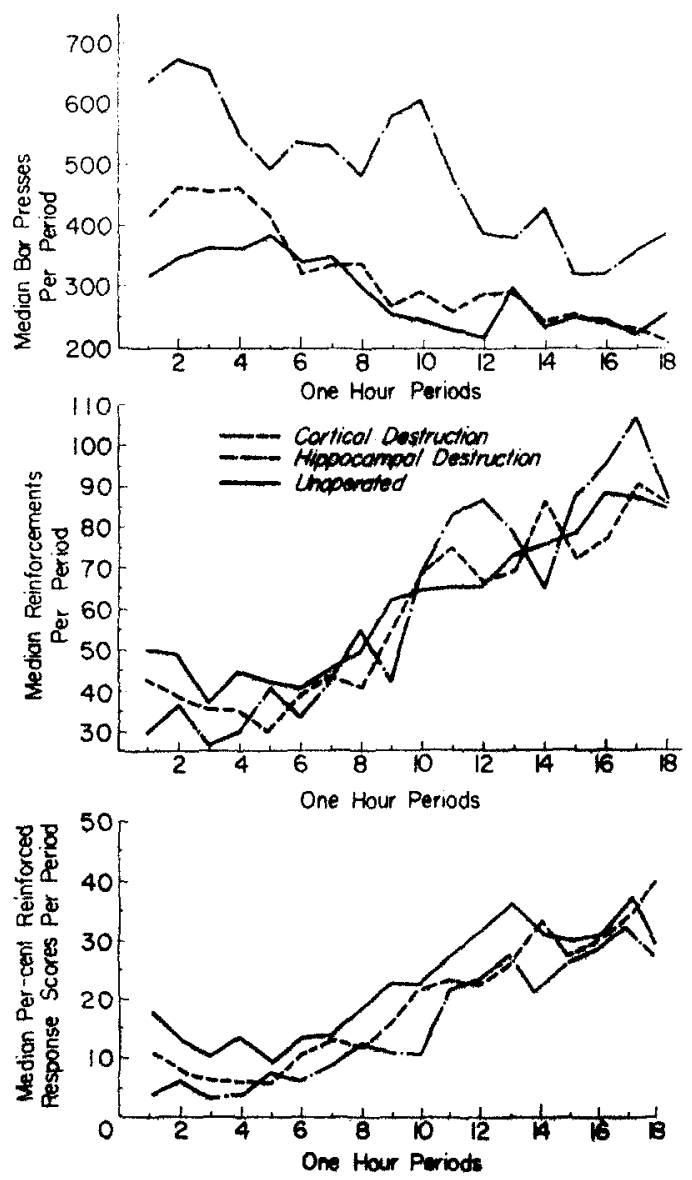

FIG. 5. Performance meanures for the animals in Condition II on DRL 20. (The animals in this Condition had no prior expertence on CRF.)
Condition Il: DRL 20 performance. The animals in this condition were given 36 consecutive sessions on the DRL 20 schedule. The scores from two $30 \mathrm{~min}$ sessions were combined into a one hr period score and the data are reported in terms of these 18 periods.

The hippocampectomized rats in this condition pushed the bar significantly more than did the cortical control and unoperated normal animals. This is presented graphically in Fig. 5. A two-way analysis of variance showed a significant effect among groups of animals ( $F$ - $9.05 ; p<0.01)$ and over DRL sessions $(F=3.81 ; p<0.01)$. There was also a significant interaction effect $(F-3.81 ; p<0.01)$. Individual comparisons showed the hippocampectomized rats to differ significantly $(F \quad \therefore 11.45 ; p<0.01)$ from the cortical control animals. There was no statistical difference between the cortical control animals and the unoperated normal rats.

Even though the hippocampectomized rats pressed more. they received as many reinforcements as the other animals in this condition. This is shown graphically in Fig. 5. A twoway analysis of variance of the number of reinforcements received by the three groups of animals showed no significant effect across groups and no significant interaction effect. There was, however, a significant effect $(F=3.32 ; p \cdot 0.01)$ over the DRL sessions.

As was done for the animals in condition 1. a per cent reinforced response score was computed for each animal for each hour period. The median per cent reinforced response scores for the three groups of animals in Condition II are plotted in Fig. 5.

The same three performance scores that were computed for the animals in Condition I were computed for each animal in Condition II. In this condition, however, the average was taken over the last 5 hour-long periods. The performance scores were not found to be significantly correlated (Pearson product-moment) with unit brain damage to the various structures mentioned in conjunction with Condition I within either the neodecorticate or hippocampectomized groups.

\section{DISCUSSION}

Observing the behavior of hippocampectomized rats in the operant chamber makes the finding that these animals are easier to shape to the bar press response than normal rats more understandable. The initial reinforcements have highly differential effects on the three groups of animals. The hippocampally lesioned rats take up a position close to the food cup after several reinforcements. The normal and neodecorticate rats, on the other hand, continue to explore the operant chamber and require many more reinforeements until they are magazine trained.

In the majority of cases, the hippocampectomized rats, once magazine trained, were easily shaped to the bar press response. This was chiefly due to the repetitive nature of much of their behavior. Once reinforced for a response, they tended to repeat this response over and over again until another reinforcement was given. In a few instances, however, the repetitive behavior of hippocampectomized animals interfered with the shaping process. Responses became so rigid that it was very difficult to use the successive approximations technique.

These behavioral observations of rats with hippocampal damage are in accord with previous experimental findings. Wickeleron and Isaacson [17] and Raphelson, Isaacson, and Douglas [13] have roported that hippocampal rats are less distractable than normal or control subjects. Since rats with 
hippocampal damage appear less distractable in certain situations, this might explain their behavior in the operant chamber.

The repetitiveness of the behavior of the hippocampectomized rats in the operant chamber is in agreement with experimental data linking this part of the limbic system to perseverative behaviors. The reports of several investigators $[6,10,11]$ seem to establish the fact that hippocampally lesioned animals are impaired in their ability to inhibit certain kinds of responses.

The superiority of the neodecorticate animals over the unoperated rats, though not statistically significant, was substantial and was obtained using both shaping measures. Observing the behavior of these animals in the operant chamber did not suggest any ready explanation for this possible superiority.

Most striking is the observation that hippocampectomized rats can master the DRL 20 schedule of reinforcement under the proper acquisition conditions. Rats in this study with extensive lesions of the hippocampus were able to respond consistently in such a manner that 50 per cent or more of their responses were reinforced ones. This mastery of the delay schedule only occurred when no experience on CRF preceded the DRL schedule. Any explanation which employs defective temporal discriminatory ability to explain the poor performance of hippocampally lesioned rats on a DRL schedule is therefore called into question.

Even though the hippocampectomized rats which were only on the DRL 20 schedule were able to achieve as many reinforced responses as the other animals, they still bar pressed significantly more than these animals. This tendency toward greater rates of responding occurred in both DRL situations -both when CRF preceded DRL and when it did not.

Clark and Isaacson [1] explained the poor performance of their hippocampal animals on the DRL 20 schedule by hypothesizing an inability to withhold the bar press response during the delay period. Jarrard [9] placed hippocampectomized rats on a VI (variable interval) schedule of reinforcement, after CRF, and found that these animals bar pressed significantly more than control or unoperated rats. He also found that rats on a VI schedule after CRF showed increased rates of responding following hippocampal destruction. Animals receiving neocortical destruction and unoperated rats did not show this increased rate of responding. Jarrard's explanation for this behavior is very similar to the one offered by Clark and Isaacson.

Extinction is a component process in the formation of the temporal discrimination required under DRL. That hippocampectomized rats show increased resistance to extinction in a linear runway has been shown $[8,12,14]$. In the present study, it could be hypothesized that those hippocampectomized rats who had long experience on CRF failed to master the DRL schedule because their greater resistance to extinction impaired their ability to inhibit the firmly established bar press response. By the same token, the performance of the hippocampally lesioned animals who were on DRL 20 from the start could be explained by hypothesizing that these animals were able to inhibit or "extinguish" bar presses during the $20 \mathrm{sec}$ delay period despite their greater resistance to extinction because the original bar press response was not well enough established in them.

One possible alternative explanation for the poor performance of the hippocampectomized rats is that such lesions cause a general increase in drive level resulting in greater activity and more bar presses. This seems unlikely in that hippocampally lesioned animals did not bar press significantly more frequently on CRF than other animals; it was only after the introduction of the DRL schedule that the increased response rate occurred. Clark and Isaacson [1] reported that their hippocampectomized rats bar pressed on CRF significantly less than control and unoperated rats. Jarrard [9] found that hippocampectomized rats did not differ from normal and control animals in the number of non-reinforced bar presses emitted before learning to bar press for food.

Furthermore, hippocampal destruction does not cause an elevation in drive level as measured in a linear runway, Raphelson, Isaacson and Douglas [14]. Douglas and Isaacson [3] reported that extensive hippocampal lesions did not affect running speed in the exercise wheel. This test is frequently used to measure drive [16] and would indicate no great change in drive level resulting from this type of lesion.

As was stated earlier, Ellen et al. [5] found that animals with damage to the hippocampus were able to acquire a DRL 20 schedule of reinforcement. This acquisition took place after experience on CRF. This is in contradiction, of course, to the present study in which hippocampectomized rats who had experience on CRF showed little or no signs of acquiring this delay schedule. Ellen et al. gave their hippocampal animals 34 fifty-min sessions of DRL 20 training. There existed the possibility, then, that had the hippocampectomized rats in Condition I been given more time in the operant chamber they would have eventually acquired the delay schedule. With this possibility in mind, these animals were given an additional 24 DRL 20 sessions after the initial 16 sessions for a total of 40 DRL sessions. At the end of this time, there was some slight improvement in performance with two of the hippocampectomized rats consistently achieving reinforcement on 25 per cent of their responses. The median per cent reinforced response scores for the last four sessions varied from 16 per cent to 19 per cent. These scores, however, are far below those which Ellen et al. report in their study for animals with hippocampal damage.

In an earlier study, Ellen and Powell [4] reported that hippocampal animals responded with fewer bar presses than control animals on a FI (fixed interval) schedule of reinforcement. The animals were introduced to this FI schedule after experience on CRF. This finding is not in accordance with the interpretation of the results of the present study. If hippocampal rats are impaired in their ability to inhibit an established bar press response, they should show greater, not lower, bar press rates on a FI schedule.

In both the Ellen et al. [5] and Ellen and Powell [4] studies, the lesions of the hippocampus were made electrolytically. The lesions in the present study were made by aspiration. There is recent evidence [3] that different patterns of behavior can result from hippocampal lesions depending upon the method used to make the lesions.

Secondly, the electrolytic hippocampal lesions in both Ellen $[4,5]$ studies were confined to the most dorsal aspects of the hippocampus. Those in this study included not only the dorsal aspects of the hippocampus; but the lateral and, in some cases, ventral portions of the hippocampus as well. Snyder and Isaacson [15] have shown for certain tasks the lateral and ventral aspects of the hippocampus of the rat must be destroyed before impairment in the ability to inhibit acquired responses is obtained.

There are, then, two factors which might account for the different behaviors of animals with hippocampal lesions reported in the various studies. Either location or amount 
of hippocampal destruction, the method by which the lesions were produced, or a combination of these two factors could be behind the differing results.

As mentioned previously, two of the hippocampectomized rats in the present study were excluded from it due to the poor quality of their lesions. One animal sustained extensive damage to the lateral septal area, the lateral geniculate nucleus, and the lateral nucleus of the thalamus. The other animal sustained damage to the lateral and the dorso-medial nuclei of the thalamus. Both of these animals were assigned to Condition II. These animals gave no indication of being able to master the delay schedule, pressing the bar at exceedingly high rates and never achieving over 20 reinforcements in any one session. It is interesting to note that the behavior of the animal with septal damage is similar to that described by Ellen $e t$ al. [5]. If the septal area is indeed involved in the mastery of this schedule of reinforcement, then, it is not surprising that the animal in the present study with lesions in the two thalamic nuclei also failed to master it. The septal area has direct connections with these thalamic nuclei and they, also, could be involved in successful performance on the DRL 20 schedule.

\section{REFERENCES}

1. Clark, C. V. and R. L. ISAACSON. Effect of bilateral hippocampal ablation on DRL performance. J. Comp. Physiol. Psychol. 59: 137-140, 1965.

2. De Groot, J. The Rat Forebrain in Stereotaxic Coordinates. Amsterdam Noord-Hollandache, 1959.

3. Douglas, R. J. and R. L. Isaacson. Hippocampal lesions and activity. Psychonom. Sci. 1: 187-188, 1964.

4. Ellen, P. and E. W. Powell. Temporal discrimination in rats with rhinencephalic lesions. Expl Neurol. 6: 538-547, 1962.

5. Ellen, P., A. S. Wilson and E. W. Powell. Septal inhibition and timing behavior in the rat. Expl Neurol. 10: 120-132, 1964

6. Isaacson, R. L., R. J. Douglas and R. Y. Moore. The effect of radical hippocampal ablation on acquisition of avoidance responses. J. Comp. Physiol. Psychol. 54: 625-628, 1961.

7. Isaacson, R. L. and W. O. Wickelgren. Hippocampal ablation and passive avoidance. Science, N.Y. 138: 1104-1106, 1962.

8. Jarrard, L. E., R. L. Isaacson and W. O. Wickelgren. Effects of hippocampal ablation and intertrial interval on runway acquisition and extinction. J. Comp. Physiol. Psychol. 57: $442-444,1964$

9. Jarrard, L. E. Hippocampal ablation and operant behavior in the rat. Psychonom. Sci. 2: 115-116, 1965.
10. Kimble, D. P. The effects of bilateral hippocampal lesions in rats. J. Comp. Physiol. Psychol. 56: 273-283, 1963.

11. Kimura, D. Effects of selective hippocampal destruction on avoidance behavior in the rat. Can. J. Psychol. 12: 213-218, 1958.

12. Niki, $H$. The effects of hippocampal ablation on the behavior of the rat. Jap. Psychol. Res. 4: 139-153, 1962.

13. Raphelson, A. C., R. L. Isaacson and R. J. Douglas. The effect of distracting stimuli on the runway performance of limbic damaged rats. Psychonom. Sci. 3: 483-487, 1965.

14. Raphelson, A. C., R. L. Isaacson and R. J. Douglas. The effect of limbic damage on the retention and performarice of a runway response. Neuropsychologia. In press.

15. Snyder, D. R. and R. L. Isaacson. The effects of large and small bilateral hippocampal lesions on two types of passive avoidance responses. Psychol. Rep. 16: 1277-1290, 1965.

16. Strong, $P$. N. Activity in the white rat as a function of apparatus and hunger. J. Comp. Physiol. Psychol. 50: 596-600, 1957.

17. Wickelgren, W. $O$. and $R$. L. Isaacson. Effect of the introduction of an irrelevant stimulus upon runway performance of the hippocampectomized rat. Nature, Lond. 200: $48 \ldots 50$ 1963. 